\title{
Cosmic Optical Background: the view from Pioneer 10/11
}

\author{
Y. Matsuoka ${ }^{1}$, N. Ienaka ${ }^{2}$, K. Kawara ${ }^{2}$, and S. Oyabu ${ }^{1}$ \\ ${ }^{1}$ Graduate School of Science, Nagoya University, Furo-cho, Chikusa-ku, Nagoya, Japan \\ ${ }^{2}$ Institute of Astronomy, The University of Tokyo, Osawa 2-21-1, Mitaka, Tokyo, Japan \\ email: matsuoka@a.phys.nagoya-u.ac.jp
}

\begin{abstract}
We present the new constraints on the cosmic optical background (COB) obtained from an analysis of the Pioneer 10/11 Imaging Photopolarimeter (IPP) data. After careful examination of data quality, the usable measurements free from the zodiacal light are integrated into sky maps at the blue $(\sim 0.44 \mu \mathrm{m})$ and red $(\sim 0.64 \mu \mathrm{m})$ bands. Accurate starlight subtraction is achieved by referring to all-sky star catalogs and a Galactic stellar population synthesis model down to 32.0 mag. We find that the residual light is separated into two components: one component shows a clear correlation with thermal $100 \mu \mathrm{m}$ brightness, while another betrays a constant level in the lowest $100 \mu \mathrm{m}$ brightness region. Presence of the second component is significant after all the uncertainties and possible residual light in the Galaxy are taken into account, thus it most likely has the extragalactic origin (i.e., the $\mathrm{COB}$ ). The derived $\mathrm{COB}$ brightness is $(1.8 \pm 0.9) \times 10^{-9}$ and $(1.2 \pm 0.9) \times 10^{-9} \mathrm{erg} \mathrm{s}^{-1} \mathrm{~cm}^{-2} \mathrm{sr}^{-1} \AA^{-1}$ at the blue and red band, respectively, or $7.9 \pm 4.0$ and $7.7 \pm 5.8 \mathrm{nW} \mathrm{m}^{-2} \mathrm{sr}^{-1}$. Based on a comparison with the integrated brightness of galaxies, we conclude that the bulk of the COB is comprised of normal galaxies which have already been resolved by the current deepest observations. There seems to be little room for contributions of other populations including "first stars" at these wavelengths. On the other hand, the first component of the IPP residual light represents the diffuse Galactic light (DGL) - scattered starlight by the interstellar dust. We derive the mean DGL-to-100 $\mu \mathrm{m}$ brightness ratios of $2.1 \times 10^{-3}$ and $4.6 \times 10^{-3}$ at the two bands, which are roughly consistent with the previous observations toward denser dust regions. Extended red emission in the diffuse interstellar medium is also confirmed.
\end{abstract}

Keywords. cosmology: observations — dark matter — diffuse radiation — dust, extinction galaxies: evolution — infrared: ISM — methods: data analysis — space vehicles

\section{Introduction}

The cosmic optical background (COB) is the optical component of the extragalactic background light, which is the integrated radiation from all light sources outside the Galaxy. Dominant contribution to the COB comes from stellar nucleosynthesis in galaxies at redshifts $z<10$, while other mechanisms such as mass accretion to super massive black holes in active galactic nuclei, gravitational collapse of stars, and particle decay can contribute to the COB brightness. As a fossil record of light production activity in the Universe, the COB conveys information on the cosmic star formation history including birth and death of Population III stars. However, robust detection of the COB has long been hampered by the extremely bright foreground emissions. While expected brightness of the COB is around $1 \mathrm{bgu} \equiv 1 \times 10^{-9} \mathrm{erg} \mathrm{s}^{-1} \mathrm{~cm}^{-2} \mathrm{sr}^{-1} \AA^{-1}$, the terrestrial airglow and the zodiacal light (ZL) are a few orders of magnitude brighter than this level at optical wavelengths (Leinert et al. 1998). The diffuse Galactic light (DGL), which refers to scattered starlight by the interstellar dust, is another but much fainter component of the diffuse light of the night sky. In order to overcome this long-standing 
problem of foreground removal, we made use of the imaging data obtained by the Imaging Photopolarimeters on board the Pioneer 10 and 11 spacecrafts (Matsuoka et al. 2011). The method and obtained results are presented here.

\section{Observations and Reductions}

The detailed description of the observations and the primary data processing can be found in, e.g., Weinberg et al. (1974) and Gordon et al. (1998). Using the Pioneer 10 IPP data collected at various heliocentric distances $R$, Hanner et al. (1974) find that the ZL brightness is below the detectable level of the instrument when the spacecraft is at beyond $R=3.26$ AU. Hence the data obtained at the larger distances are most suitable for an analyses of diffuse radiations outside the ZL clouds.

We performed thorough cleaning of the data by removing those with negative flux record, possible contamination of the scattered sunlight, abnormal values of fluxes and/or colors, and so on. Then all the good-quality data were integrated into a single sky map at each of the IPP blue $\left(B_{\mathrm{IPP}}\right)$ and red $\left(R_{\mathrm{IPP}}\right)$ bands, using a similar algorithm to 1 st iteration of the "maximum correlation method" (Aumann et al. 1990). The final maps have the angular resolution of $\sim 0^{\circ} .7$. The possible systematic uncertainties were estimated by dividing the data into various subgroups with several criteria such as spacecrafts and heliocentric distances and comparing the sky maps created from each of them. The full description of the data reduction process is given in Matsuoka et al. (2011)

\section{Galactic Light}

Outside the detectable ZL clouds, the dominant brightness component incident on the IPPs is Galactic starlight. Since the contribution of brightest stars has already been subtracted when the archival data were created, we had to subtract the contribution of fainter stars from the IPP measurements. Integrated brightness of relatively bright $(V<11 \mathrm{mag})$ and faint $(V>11 \mathrm{mag})$ stars were calculated from the Tycho-2 Catalog (Høg et al. 2000) and the Hubble Space Telescope (HST) Guide Star Catalog II (GSCII) version 2.3 (Lasker et al. 2008). Furthermore, the contributions of stars even fainter than the GSC-II detection limits were estimated, down to 32.0 mag, using a star count model provided by a stellar population synthesis code TRILEGAL (Girardi et al. 2005). Transformations of stellar magnitudes in the different passband systems were derived using the Bruzual-Persson-Gunn-Stryker (BPGS) stellar spectral atlas. We integrated the starlight-subtracted IPP brightness into sky maps with the same algorithm as described above, which gave us the "diffuse emission maps" comprised of diffuse Galactic and extragalactic emission components.

The DGL is attributed to dust and gas particles in the interstellar medium (ISM). Dominant contribution to the optical DGL comes from scattering of the interstellar radiation field (ISRF) by the dust. We estimate the DGL contribution to IPP diffuse emission brightness by separating out the component which correlates with the diffuse Galactic far-IR emission. The good correlation between the DGL and far-infrared (IR) emission brightness is a natural result from the fact that both emissions are caused by the interstellar dust exposed to the ISRF, and is actually observed in several regions (compiled in Bernstein et al. 2002). With the DGL-to-100 $\mu \mathrm{m}$ brightness ratio $a_{\mathrm{d}}$, the IPP diffuse emission brightness $S^{\text {diffuse }}$ can be written as:

$$
\begin{aligned}
S^{\text {diffuse }} & =S^{\mathrm{DGL}}+S^{\mathrm{COB}} \\
& =a_{\mathrm{d}}\left(S_{100 \mu \mathrm{m}}^{\text {diffuse }}-S_{100 \mu \mathrm{m}}^{\mathrm{CIB}}\right)+S^{\mathrm{COB}}
\end{aligned}
$$


where $S^{\mathrm{DGL}}$ and $S^{\mathrm{COB}}$ are the DGL and COB brightness at the IPP bands, and $S_{100 \mu \mathrm{m}}^{\text {diffuse }}$ is diffuse $100 \mu \mathrm{m}$ brightness observed outside the ZL clouds. The cosmic infrared background (CIB) component $S_{100 \mu \mathrm{m}}^{\mathrm{CIB}}$ is subtracted from $S_{100 \mu \mathrm{m}}^{\text {diffuse }}$ in the above equation, leaving only Galactic component.

\section{Results and Discussion}

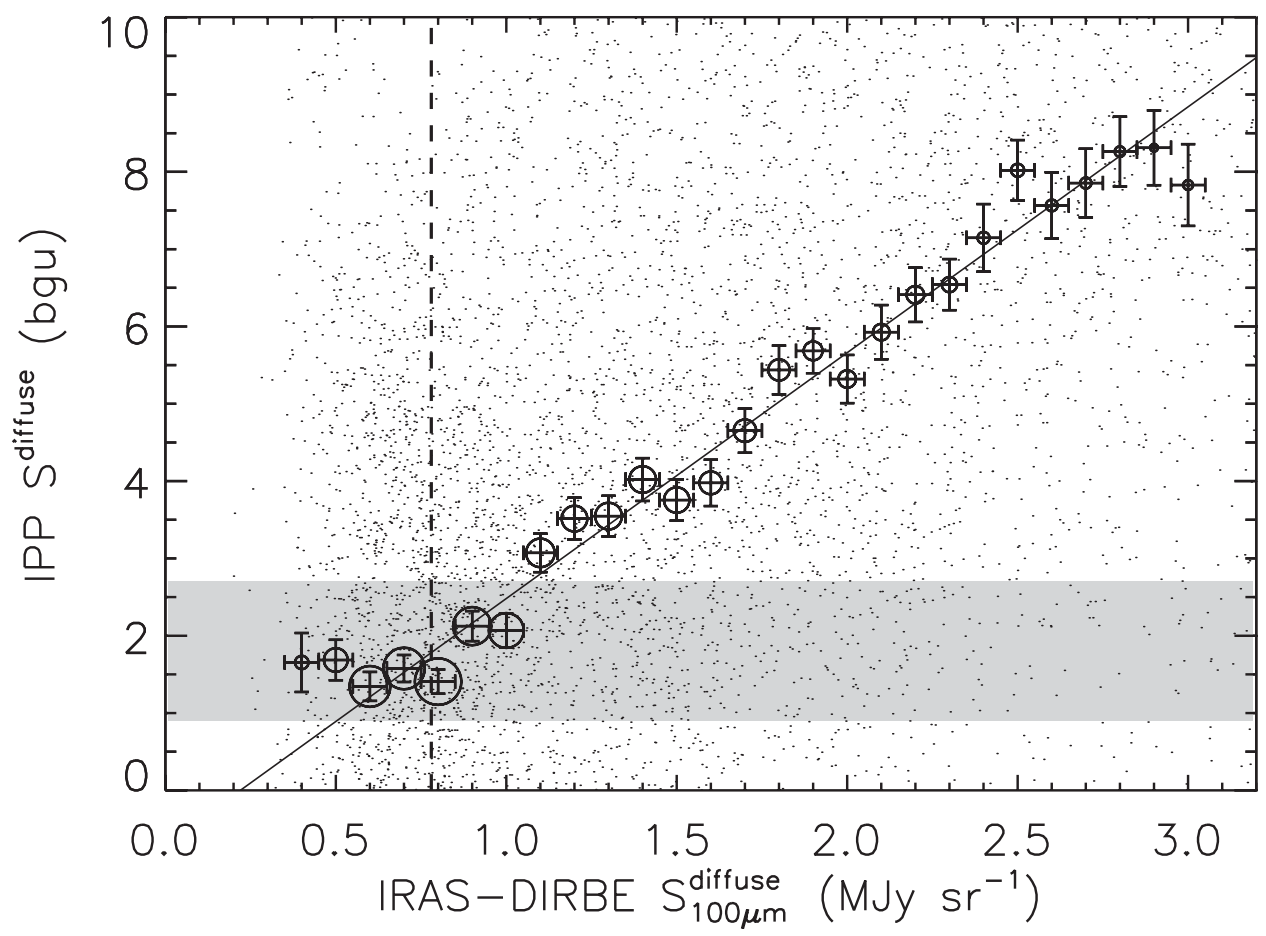

Figure 1. Observed IPP diffuse emission brightness $S^{\text {diffuse }}$ at $B_{\text {IPP }}$ versus the diffuse $100 \mu \mathrm{m}$ brightness $S_{100 \mu m}^{\text {diffuse }}(d o t s)$. The open circles and error bars represent mean values of $S^{\text {diffuse }}$ and their errors in the $S_{100 \mu m}^{\text {diffuse }}$ bins. The sizes of the circles are proportional to numbers of the data points in the bins. The solid line shows the regression line at $S_{100 \mu m}^{\text {diffuse }}>1.0 \mathrm{MJr} \mathrm{sr}^{-1}$, while the dashed line shows the CIB brightness $S_{100 \mu m}^{\mathrm{CIB}}$ reported by Lagache et al. $(2000)$. The shaded area shows $1 \sigma$ confidence interval of our final COB estimate.

We show the measured IPP diffuse emission brightness $S^{\text {diffuse }}$ versus the diffuse $100 \mu \mathrm{m}$ brightness $S_{100 \mu \mathrm{m}}^{\text {diffuse }}$ outside the ZL clouds in Figure 1, at $B_{\mathrm{IPP}}$ band as an example. Our analysis focuses on the lowest brightness region with $S_{100 \mu \mathrm{m}}^{\text {diffuse }}<3.0 \mathrm{MJy} \mathrm{sr}^{-1}$ and with the IPP map coverage at the Galactic latitudes $|b|>35^{\circ}$, which corresponds to about a quarter of the whole sky. The diffuse $100 \mu \mathrm{m}$ brightness is taken from Schlegel et al. (1998). We clearly detect the linear correlations between $S^{\text {diffuse }}$ and $S_{100 \mu \mathrm{m}}^{\text {diffuse }}$ at the large $S_{100 \mu \mathrm{m}}^{\text {diffuse }}$, and more importantly, the flattening of these relations at $S_{100 \mu \mathrm{m}}^{\text {diffuse }}<0.8 \mathrm{MJy} \mathrm{sr}^{-1}$ in the both IPP bands. The inflection points are in very good agreement with the CIB brightness reported by Lagache et al. (2000), which had been predicted in a Monte-Carlo simulation (Matsuoka et al. 2011). Our estimates of the COB brightness are derived as the $S^{\text {diffuse }}$ values on the observed $S^{\text {diffuse }}-S_{100 \mu \mathrm{m}}^{\text {diffuse }}$ linear correlations made by the DGL, at the point where $100 \mu \mathrm{m}$ brightness equals to the CIB. Taking all the uncertainties in the measurement and data reduction processes into account, the obtained results are 
$S^{\mathrm{COB}}=1.8 \pm 0.9$ and $1.2 \pm 0.9 \mathrm{bgu}$, or $7.9 \pm 4.0$ and $7.7 \pm 5.8 \mathrm{nW} \mathrm{m}^{-2} \mathrm{sr}^{-1}$, at $B_{\mathrm{IPP}}$ and $R_{\mathrm{IPP}}$ bands, respectively.

We compile the current measurements of the cosmic background and the integrated brightness of galaxies at ultraviolet, optical, and near-IR wavelengths in Figure 2. In contrast to Bernstein (2007), our new results of the COB brightness are mildly larger than and consistent within $1 \sigma$ uncertainty with the integrated brightness of galaxies measured in the Hubble deep field (HDF; Madau \& Pozzetti 2000). They are on the smooth extension of the upper limits found by Aharonian et al. (2006). With our best estimates, approximately $60 \%$ and $90 \%$ of the COB have already been resolved into discrete galaxies in the $\mathrm{HDF}$ at $0.44 \mu \mathrm{m}\left(B_{\mathrm{IPP}}\right)$ and $0.64 \mu \mathrm{m}\left(R_{\mathrm{IPP}}\right)$, respectively. On the other hand, Totani et al. (2001) demonstrate that $60-90 \%$ and $80-100 \%$ of the total light from galaxies have been resolved at 0.45 and $0.61 \mu \mathrm{m}$. The above facts indicate that bulk of the $\mathrm{COB}$ are comprised of normal galaxies, and there are little room for contributions of other populations at these wavelengths.

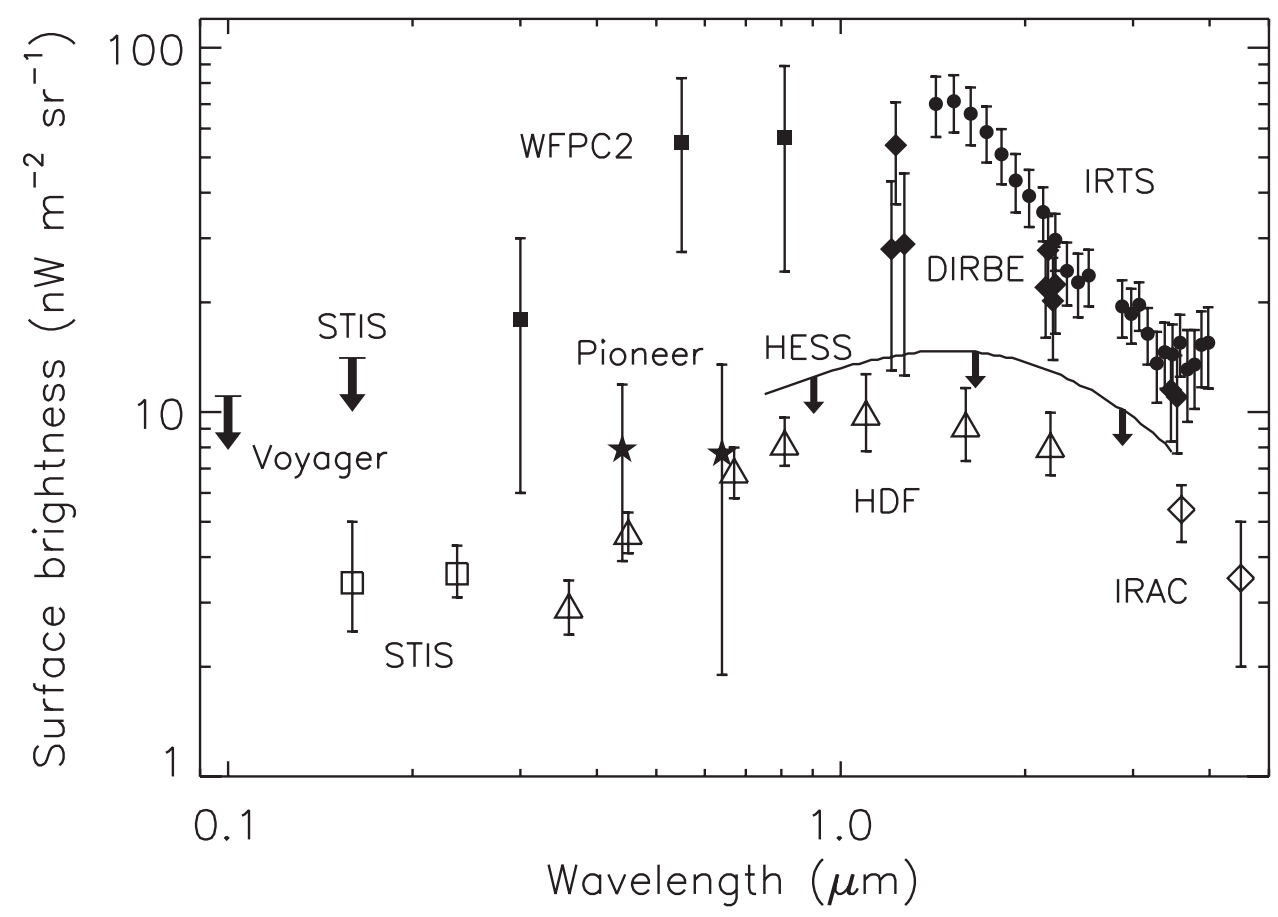

Figure 2. Current measurements of the cosmic background (filled symbols) and the integrated brightness of galaxies (open symbols). At optical wavelengths, squares and stars represent the results by Bernstein (2007) and Matsuoka et al. (2011). The solid line with arrows between 0.8 and $4 \mu \mathrm{m}$ represent the upper limits reported by Aharonian et al. (2006). The integrated brightness of galaxies at the corresponding wavelengths are given by Madau \& Pozzetti (2000) (triangles). Further details of this figure are described in Matsuoka et al. (2011).

\section{References}

Aharonian, F., Akhperjanian, A. G., Bazer-Bachi, A. R., et al. 2006, Nature, 440, 1018

Aumann, H. H., Fowler, J. W., \& Melnyk, M. 1990, AJ, 99, 1674

Bernstein, R. A. 2007, ApJ, 666, 663

Bernstein, R. A., Freedman, W. L., \& Madore, B. F. 2002, ApJ, 571, 56 
Girardi, L., Groenewegen, M. A. T., Hatziminaoglou, E., \& da Costa, L. 2005, A $\mathscr{E} A, 436,895$ Gordon, K. D., Witt, A. N., \& Friedmann, B. C. 1998, ApJ, 498, 522

Hanner, M. S., Weinberg, J. L., Deshields, L. M., II, Green, B. A. \& Toller, G. N. 1974, J. Geophys. Res., 79, 3671

$\mathrm{H} \varnothing \mathrm{g}$, E., Fabricius, C., Makarov, V. V., et al. 2000, A\&̊A, 355, L27

Lagache, G., Haffner, L. M., Reynolds, R. J., \& Tufte, S. L. 2000, A\&A, 354, 247

Lasker, B. M., Lattanzi, M. G., McLean, B. J., et al. 2008, AJ, 136, 735

Leinert, C., Bowyer, S., Haikala, L. K., et al. 1998, A\&AS, 127, 1

Madau, P. \& Pozzetti, L. 2000, MNRAS, 312, L9

Matsuoka, Y., Ienaka, N., Kawara, K., \& Oyabu, S. 2011, ApJ, 736, 119

Schlegel, D. J., Finkbeiner, D. P., \& Davis, M. 1998, ApJ, 500, 525

Totani, T., Yoshii, Y., Iwamuro, F., Maihara, T., \& Motohara, K. 2001, ApJ, 550, L137

Weinberg, J. L., Hanner, M. S., Beeson, D. E., Deshields, L. M., II \& Green, B. A. 1974, J. Geophys. Res., 79, 3665

\section{Discussion}

Matilla: This has been a very careful re-analysis of the valuable Pioneer 10/11 background starlight data base. Still, I have two questions on the systematic errors which are crucial for the final result:

Firstly, the IPP gives systematic errors of $8 \%$ (Blue) and $13 \%$ (Red), but never less than $4 \mathrm{~S}_{10}(\mathrm{~V})_{G_{2 V}}$. You announce and use, instead of these, ten times smaller errors of $1 \%$ (or 0.4 background). Can you defend these small systematic errors?

Secondly, the IRAS $100 \mu \mathrm{m}$ surface brightness values, even after rescaling with COBE/DIRBE, do not have an accurate zero point better than $\sim 1 \mathrm{MJy} /$ sterad (perhaps). How does this uncertainty influence your EBL value derived from the I (optical) diffuse vs IRAS $100 \mu \mathrm{m}$ diagram?

MATSUOKA: With regard to the first question, we have performed the direct comparison between the brightness measured by the IPPs and the independent star catalogues, and found systematic differences of a few percent. I wonder whether the level of $\sim 10 \%$ systematic errors you mentioned apply to the lowest brightness regions on which our analysis is based.

With regard to the second point, indeed the CIB brightness of Lagache et al. (2000), based on the same COBE-DIRBE calibration as the Schlegel et al. (1998) $100 \mu \mathrm{m}$ map we used, is reported to be affected by the residual zodiacal light (ZL). But our results suggest that the similar amount of residual ZL is present in the Schlegel map, and this kind of uncertainty cancels out in our method (see our paper). In any case, we expect to have more reliable results when future far-IR maps, e.g. from the AKARI survey, are available. 